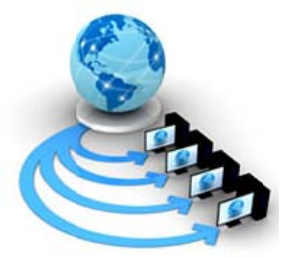

Volume 9, No. 2, March-April 2018

International Journal of Advanced Research in Computer Science

REVIEW ARTICLE

\author{
Available Online at www.ijarcs.info
}

\title{
A QUALITATIVE ANALYSIS OFWIRELESS ROBOTIC ARMS
}

\author{
Akash Bajoria, \\ Department of Computer Science \\ Mukesh Patel School of Technology, Management and \\ Engineering, NMIMS \\ Mumbai, India
}

\author{
Soham Bhatnagar, \\ Department of Computer Science \\ Mukesh Patel School of Technology, Management and \\ Engineering, NMIMS \\ Mumbai, India
}

\author{
Jaideep Bhide, \\ Department of Computer Science \\ Mukesh Patel School of Technology, \\ Management and Engineering, NMIMS \\ Mumbai, India
}

\author{
Sidhant Birla, \\ Department of Computer Science \\ Mukesh Patel School of Technology, Management and \\ Engineering, NMIMS \\ Mumbai, India
}

\author{
Artika Singh, \\ Department of Computer Science \\ Mukesh Patel School of Technology, Management and \\ Engineering, NMIMS \\ Mumbai, India
}

\begin{abstract}
The purpose of this paper is to determine the qualitative advantages and the design of a robotic arm. From the perspective of a client, the research investigated the positive and negative aspects of the current robots available on the online marketplace, and how the self-designed arm discussed in the paper shall compares with these. The research techniques were mixed qualitative methods that provided cross-examination and included research, observations, and case studies. The analysis used triangulation and resulted in the discovery of a vast difference in pricing and features of current robots, which provided a need for a low cost yet efficient robot, unavailable in the existing scenario. The research also indicated that robotic arms are increasingly being used in home automation as well, apart from the existing industrial automation clientele. The paper also discusses the self-designed robot's capabilities, in comparison to the existing robotic arms.
\end{abstract}

Keywords: Android Application; Micro-Controller; Robotic Arm; Wireless Control.

\section{INTRODUCTION}

A robotic arm is a type of mechanical arm, usually programmable, with similar functions to a human arm, the arm may be the sum total of the mechanism or may be part of a more complex robot. The links of such a manipulator are connected by joints allowing either rotational motion (such as in an articulated robot) or translational (linear) displacement. The links of the manipulator can be considered to form a kinematic chain. The terminus of the kinematic chain of the manipulator is called the end effector and it is analogous to the human hand. Area of concern and the reasons why development of robotic arm became necessary are - human errors, handling errors, hazardous substance handling, etc.

Since early 2015, the percussive mechanism in the drill that helps chisel into rock has had an intermittent electrical short. In other recent developments, we have haptic and remotecontrol arm technologies used extensively in space for carrying, holding, adjusting and precision of equipment. We also have growing use of such arms in industries to avoid dangerous situation where any control faults due to human error can cause fatal losses. Completely automated industries are also being developed so as to reduce human workload.
We aim to develop a robotic arm that performs all the available combinations of movement in the current market and more within a smaller cost frame, aiming to do so after already having conducted a market survey and discovering that all available options in this area are either way too expensive or are not developed enough to sink money into. Our motivation for taking up researching of robotic arms was our zeal to help in providing ease and comfort in hospitals for patients who are suffering through weakness and cannot perform simple actions. Simply put, we aim to make the lives of thousands simpler, and hopefully, better. We have reviewed a few research papers and taken ideas as well as drawn conclusions from the same, in doing so we have decided the type of robotic arm we intend to design.

\section{SURVEY OF VARIOUS ROBOTIC ARMS}

\section{A. Research Paper 1 [1]}

The paper discusses the implementation of using speech as primary input for the functioning of a wireless pick and place robot. The method used is speaker independent, and understands 8 commands such as up, down, pick, place, etc. The ZigBee Module is essential to the functioning of this speech input. This module is used to transmit the different voice commands or remote control made by the user 
(encoded in the form of 4-bit digital data) wirelessly to the receiver, which decodes the received 4-bit digital data and according to which the arm, gripper and platform moves.

The robot aims to be user friendly in the social context, with user independent speech as the combining fragment for its popularity. However, the robot can only function in controlled environments, i.e., exempt on any noise interfering with the voice commands. The presence of persistent noise results in the robot not being able to differentiate between sounds and hence, not recognizing the commands. The build itself is quite poor, with no cladding provided to any parts, and thus is extremely vulnerable to wear and tear.

\section{Advantages}

- Low Cost

-Appealing and the most user-friendly method of input

\section{Disadvantages}

- No cladding over the robot makes it vulnerable to damage

- The voice commands do not specify a unit of movement in a direction, hence the robot is not accurate enough

- Noise in the surrounding environment makes the input redundant as the robot cannot infer it

- Method of communication limits its usage only to a range of good audibility

\section{B. Research Paper 2 [2]}

The robotic arm discussed in this paper is made from acrylic sheets which have been laser cut. The motors are mounted and connected the Arduino board allowing the robot to move. The robot has 4 degrees of freedom two about the base, one about the elbow joint and one about the grabber. The board can now be programmed to move the arm. Additional sensors can be added for more functionality. The robot can be controlled via a remote.

\section{Advantages}

-Modular design

- Low Cost

- Reprogrammable

-4 Degrees of freedom

\section{Disadvantages}

- The wires are exposed leading to the robot being fragile

- The robot is requiring some knowledge about soldering for adding additional modules

\section{Research Paper 3 [3]}

This paper proposes control using haptic glove control.

\section{Design}

A. Haptic gloves: This part is mainly used for controlling the robot arm. The design of the gloves is completely same as the human hand. But here we are discussing potentiometer-based gloves. The potentiometer used to control the position of the robot arm, these are connected to each and every finger of gloves and when we move the hand same output will be done by the robot arm. This sensor is the device that measures a physical touch into an electrical signal. MEMS (Micro Electro-Mechanical Systems) potentiometer is one frequent sensor can be used [4].

B. Robot arm: The body complete robot arm is fixed on the base. The hand is made up of acrylic fiber. The pick and place capacity totally depend upon the kind of motors used.

C. Microcontroller AVR 8535: Every automated system has got microcontroller as base. This paper used AVR microcontroller. The AVR 8535 microcontroller has high performance and low cost. It is 8-bit microcontroller and it has a power consumption capacity with best speed. This is present on both side i.e. glove and robot arm side.

D. RF transceiver module: This is acting as transceiver. It has got CC 2500 chip which $2.4 \mathrm{GHz}$ transceiver.

\section{E. ST 3654 -CC 2500 ZigBee Based Transceiver Module:}

This module is installed at both the ends for input and out of signal. ZigBee module is used for two-way communications.

F. Zigbee Module: Zigbee is the most promising wireless communication technologies because of it high reliability, good data rate, easy to use and low cost. Zigbee is the name for a collection of high-level communication protocols for devices based on the IEEE802.15.4 standard. Zigbee is an $\mathrm{RF}$ (radio frequency) communication standard that makes it very simple to transmit digital information between devices. Zigbee modules are inexpensive components which integrate an antenna, amplifier, transmitter/receiver, and circuitry that allows you to send and receive data between the Zigbee and a microcontroller over a standard serial connection. Zigbee modules are perfect for applications like building a remotecontrol unit for your robot, or allowing your robot to wirelessly transmit data such as sensor readings and internal states. Zigbee is a protocol stack based on the 802.15.4 wireless network communication standard. It is low-cost, low-power and all-in-all perfectly suited for low-bandwidth communicationneeds. The bandwidth is on the order of tens to hundreds of kilobits per second, and the range is up to about a kilometer, depending on equipment.

\section{Motors}

A. Motor driver Section: The microcontroller receives the code for left, right, up, down of robot from PC at port 1. It checks the code and according to that provides the data to motor on port o. To run the motor, we have used L298 motor driver IC. Two motors on the left side of the robot are connected in parallel combination \& other two in the right side are connected in parallel combination. Apart from this motor such as B. IC L298 motor is also used.

\section{Advantages}

- Gives the real-time action with objects

- It works continuously and won't take any rest

- Precise control of tool during operation

- Controlling is also very easy as it is controlled by haptic gloves 


\section{Disadvantages}

- Implementation cost of Haptic technology is very expensive

- Debugging issues

- Haptic applications can be very complex design and it's also required specialized hardware and accordingly processing power

\section{Research Paper 4 [4]}

The paper presents the design of a robotic arm with 3 Degrees of Freedom. The design is influenced by a number aspect, such as geometry of the manipulator, dynamics, structural characteristics of the manipulators and actuator characteristics. The arm resembles a human arm, with the stationary part of the arm being the shoulder. The arm is designed such that it is slender. This is done to reduce weight, which is crucial in reducing power consumption during its operation. The end of the arm has a carry endeffector, which takes the role of a gripping mechanism.

The motion of the arm is given by kinetic modelling. To perform kinetic modeling of the robotic manipulators the Denavit-Hartenberg convention and the screw theory technique is used. The method involved in using the cartesian coordinates of the arm link to find the desired joint space for generation of trajectory. The force and the torque of the arm is determined by the Euler-Lagrange formula. The analytic simulations of the results were done on RoboAnalyzer software.

\section{Advantages}

- The robotic arm is designed for joint fault tolerance. The arms are slender thereby reducing the weight of the joints and also the overall power consumption during operation

- The arm also uses efficient mathematical formula to calculate and determine accurate movement of the robot

\section{Disadvantages}

- The robot although accurate in movement does not have enough strength to carry objects that are big and heavy

\section{E. Research Paper 5 [5]}

The paper discusses an android application that provides an interface for controlling the arm via two mechanisms; Smartphone Tilt and Remote, using either of the wireless networks, a boosted- Bluetooth or Wi-Fi. The paper focuses on methods of control and communication at length rather than the construction of a robotic arm.

\section{Wireless Communication Mechanisms}

A. Wi-Fi:

B. The arm uses an ESP8266 Wi-Fi module with integrated TCP/IP protocol stack that can give any microcontroller access to an open WIFI network.

\section{B. Bluetooth:}

The arm uses a HC-05 module which is an easy to use Bluetooth SPP (Serial Port Protocol) module. It usesCSR Bluecore 04-External single chip Bluetooth system with CMOS technology and with AFH (Adaptive Frequency Hopping Feature), enabling the Bluetooth to work over much larger distances than usual.

\section{Control Mechanisms}

A. Phone Tilt:

B. The phone tilt control works on the change in orientation of the phone which can be measured by the inbuilt accelerometer in the phone; the change of axis detected by the accelerometer will be transmitted via Bluetooth or Wifi to the microcontroller.

\section{Remote Control:}

D. A simple interface is made in the android application to transfer commands to the arm; using touch buttons. The command can be transmitted to the microcontroller via Wifi or Bluetooth.

\section{Advantages}

- Offers two modes of communication for varied distances

- Uses an Android application to control the robot

-User friendly, easy to use

\section{Disadvantages}

-Quite expensive

-Only at prototype stage

\section{COST AND VERDICT}

We performed a thorough market survey on the pick and place robots available for the public (Robokits, Amazon India, Robotworx- the leading online retailers of pick and place robots). [6] The high-end pick and place robots available in the market are have great features such as 6 degrees of freedom, high picking strength, and a combination of soft and hard grips. These robots though, cost upwards of 25,000 and are not affordable for large masses of the public. The cheapest ones, starting at 7,000 , have extremely few features such as 1 or 2 degrees of freedom, no wireless connectivity, poor build quality, and low to none reprogramming ability. We aim to bridge the gap between the features provided by the low end and highend robots, and still cater to the market of the masses. We estimated our cost of production to be 9,000 , which fits perfectly into the low to medium cost bracket for robotic arms, while housing features found nowhere in the same price bracket, and develop it in accordance with the highest safety standards. 
TABLE I. RESEARCH OF THE BENEFICIAL ABILITIES FROM THE PAPERS

\begin{tabular}{|c|c|c|c|c|c|c|}
\hline $\begin{array}{l}\text { Sr } \\
\text { No } \\
\text {. }\end{array}$ & $\begin{array}{l}\text { Parameter } \\
\text { s }\end{array}$ & $\begin{array}{l}\text { Research Paper } \\
1\end{array}$ & $\begin{array}{l}\text { Research Paper } \\
2\end{array}$ & $\begin{array}{l}\text { Research Paper } \\
3\end{array}$ & $\begin{array}{l}\text { Research Paper } \\
4\end{array}$ & $\begin{array}{l}\text { Research Paper } \\
5\end{array}$ \\
\hline 1 & $\begin{array}{l}\text { Degrees of } \\
\text { Freedom }\end{array}$ & 3 & 4 & 3 & 3 & 3 \\
\hline 2 & Build & $\begin{array}{l}\text { Extremely low cost, } \\
\text { no external cladding } \\
\text { to protect robot, thus } \\
\text { prone to damage. }\end{array}$ & $\begin{array}{l}\text { Low cost, and } \\
\text { exposed wiring, } \\
\text { hence prone to } \\
\text { damage. }\end{array}$ & $\begin{array}{l}\text { Built with a durable } \\
\text { outer cast that can } \\
\text { withstand wear and } \\
\text { tear, equipped with } \\
\text { two grippers; hard } \\
\text { and soft. }\end{array}$ & $\begin{array}{l}\text { Lost cost build, that } \\
\text { sacrifices strength } \\
\text { for cost. }\end{array}$ & $\begin{array}{l}\text { The prototype } \\
\text { created was a low } \\
\text { cost model, with } \\
\text { cladding at the joints }\end{array}$ \\
\hline 3 & $\begin{array}{l}\text { Method of } \\
\text { Control }\end{array}$ & $\begin{array}{l}\text { Voice Control, } \\
\text { recognises } \\
\text { keywords } \\
\text { independent of the } \\
\text { user }\end{array}$ & Remote control & $\begin{array}{l}\text { Haptic } \\
\text { controlled }\end{array}$ & Computer software & $\begin{array}{l}\text { Application control, } \\
\text { phone tilt control }\end{array}$ \\
\hline 4 & $\begin{array}{l}\text { Method of } \\
\text { Communicatio } \\
\mathrm{n}\end{array}$ & $\begin{array}{lr}\text { Voice } & \text { processing } \\
\text { unit, } & \text { Zigbee } \\
\text { Transceiver } & \end{array}$ & $\begin{array}{l}\text { Bluetooth or Infra } \\
\text { Red }\end{array}$ & $\begin{array}{l}\text { Bluetooth or Infra } \\
\text { Red }\end{array}$ & LAN & $\begin{array}{lr}\text { Wi-Fi, } & \text { Bluetooth } \\
\text { with } & \text { Adaptive } \\
\text { Frequency } & \text { Hopping } \\
\text { Feature } & \end{array}$ \\
\hline 5 & Cost Bracket & Extremely Low & Low & Medium & Extremely low & High \\
\hline 6 & Findings & $\begin{array}{l}\text { The build quality of } \\
\text { the robot and the } \\
\text { method of control } \\
\text { are the biggest } \\
\text { drawbacks to the } \\
\text { robotic arm. The } \\
\text { build is too poor to } \\
\text { be durable for day to } \\
\text { day activities, and } \\
\text { the method of } \\
\text { control is not reliable } \\
\text { in uncontrolled } \\
\text { environments }\end{array}$ & $\begin{array}{l}\text { The robot assembly } \\
\text { is relatively simple } \\
\text { and does not require } \\
\text { any skilled labor. A } \\
\text { major drawback of } \\
\text { this robot is that the } \\
\text { range of the wireless } \\
\text { communication is } \\
\text { very small thus the } \\
\text { user must be in the } \\
\text { same room as the } \\
\text { robot to control it. }\end{array}$ & $\begin{array}{l}\text { The robot assembly } \\
\text { isn't so simple and } \\
\text { requires delicate } \\
\text { skilled labour. The } \\
\text { functions that can be } \\
\text { included are many. } \\
\text { Major drawback is } \\
\text { the connection range } \\
\text { and cost required for } \\
\text { this technology at } \\
\text { this time. }\end{array}$ & $\begin{array}{l}\text { The robotic arm is } \\
\text { designed for joint } \\
\text { fault tolerance. The } \\
\text { major drawback of } \\
\text { the arm is the } \\
\text { slender design that } \\
\text { makes the arm } \\
\text { unable to lift heavy } \\
\text { objects. }\end{array}$ & $\begin{array}{l}\text { The robotic arm } \\
\text { discussed is only at } \\
\text { the prototype stage, } \\
\text { and still is the } \\
\text { closest to the arm we } \\
\text { intend to design. } \\
\text { Improvements on } \\
\text { the build quality and } \\
\text { degrees of freedom } \\
\text { is necessary, while } \\
\text { regulating the costs. } \\
\text { This shall serve as } \\
\text { the blueprint for the } \\
\text { design. }\end{array}$ \\
\hline
\end{tabular}

Key: -Indicates properties of the specific robotic arm that would be most useful for the indigenous robotic arm

\section{CONCLUSION}

The automation revolution, be it home or industrial, is driving up the demand for such robots that make human life easier, and in turn smarter. Robotic arms are used in a variety of avenues such as in manufacturing, research, and home, industrial automation. Pick and place robots can be used for precise movements, handling toxic items, working in environments that are unsuitable for humans, among various other applications. Home applications of the pick and place robot are numerous, especially for differently abled people. This helping hand can be helpful in performing various daily tasks, which might not be an ease in normal circumstances. [7] Thus, pick and place robots need to be affordable and yet guarantee to provide a service that is exemplary, which is what we shall strive to achieve. The arm we intend to produce would have 4 Degrees of Freedom, would be controlled wirelessly from a distance using an Android device acting as a remote control, through an indigenous application. The arm would have varying grip strengths (hard, soft) to be able to pick up and place delicate as well a sturdy object. The robotic arm can be upgraded later on to accept inputs in different languages making it more accessible to the public.

\section{ACKNOWLEDGMENT}

Acknowledgements are dedicated to our mentor Prof. Artika Singh and our course coordinator Prof. Shubha Puthran for their guidance and collaboration in this work, to Mukesh Patel School of Technology Management and Engineering (MPSTME) for the support and allowing us to take up this project.

\section{REFERENCES}

[1] R. ASWINBALAJI, A. ARUNRAJA; "WIRELESS VOICE CONTROLLED ROBOTICS ARM”, ISSN: 0976-1353, Volume 12, Issue 4, 4th February 2015, International Journal of Emerging Technology in Computer Science \& Electronics (IJETCSE), pp. 33-38.

[2] Eric Ravenscraft, "BUILD A KICKASS ROBOT ARM: THE PERFECT ARDUINO PROJECT FOR BEGINNERS", URL: https://lifehacker.com/build-akickass-robot-arm-the-perfect-arduino-project-1700643747, visited $4^{\text {th }}$ April 2015. 
[3] Varalakshmi B D, Abhilasha Pachauri, Thriveni J, Venugopal $\mathrm{K} \mathrm{R}$, and Patnaik L M, "MEMS SENSORS CONTROLLED HAPTIC FOREFINGER ROBOTIC AID”, Vol. 5, Issue 10, October 2014, International Journal of Advanced Research in Engineering and Technology (IJARET),pp. 45- 54.

[4] Ramish, S. B. Hussain and F. Kanwal, "Design of a 3 DoF robotic arm," Dublin, 2016, 2016 Sixth International Conference on Innovative Computing Technology (INTECH), pp. 145-149.

[5] A. N. Reganti, S. Ananthapalli, B. C. V. Mohan, R. Mounica and V. K. Mittal, "Mobile Quad-Controlled Wireless
Robotic Arm,” Delhi, 2016,2016 IEEE 1st International Conference on Power Electronics, Intelligent Control and Energy Systems (ICPEICES), pp. 1-5.

[6] Tarun Agarwal, "How to Make a Robotic ArmalongwithitsWorking",URL:Https://www.edgefx.in/ho w-to-make-robotic-arm-alongworking/, 2015.

[7] Vansjaliya Kinjal, Prof. Tarun R. Dholariya, Dr. Charmy Patel, "Multidimensional Motion Control of Robotic Arm",Issue 2,Vol. 3, February 2015, International Journal of Innovative Research in Computer and Communication Engineering (IJIRCCE), pp. 893-900. 